\section{Correlação entre instrumentos de qualidade de vida relacionada à saúde e independência funcional em idosos com insuficiência cardíaca}

\author{
Correlation between instruments for measuring \\ health-related quality of life and functional \\ independence in elderly with heart failure
}

\footnotetext{
${ }^{1}$ Faculdade de Ciências Médicas, Pontifícia Universidade Católica de São Paulo, Sorocaba, Brasil. 2 Faculdade de Ciências Médicas, Universidade Estadual de Campinas, Campinas, Brasil.

Correspondência F. A. A. Scattolin Departamento de Enfermagem, Faculdade de Ciências Médicas, Pontifícia Universidade Católica de São Paulo.

Pça. Dr. José Ermírio de Moraes 290, Sorocaba, SP 18030-230, Brasil. fscattolin@pucsp.br
}

\begin{abstract}
The main purpose of this study was to identify the correlation between health-related quality of life and the Functional Independence Measure (FIM) in elderly patients with heart failure. 146 elderly outpatients completed the Minnesota Living with Heart Failure Questionnaire (LHFQ) and the FIM. Spearman correlation coefficient was used to identify correlations between health-related quality of life and FIM, and between them and New York Heart Association (NYHA) class. Statistically significant moderate correlations were found between physical and emotional LHFQ and total FIM and motor subscale. The results show that quality of life and functional independence are correlated concepts influenced by NYHA class and suggest that measures to promote functional independence (especially in the physical domain) can improve health-related quality of life in elderly patients with heart failure. Further research should include a larger sample and older patients with greater disease severity in order to verify the reproducibility of these findings.
\end{abstract}

Congestive Heart Failure; Activities Daily Living; Quality of Life; Elderly
Fátima Ayres de Araújo Scattolin 1

Maria José D'Elboux Diogo 2

Roberta Cunha Rodrigues Colombo 2

\section{Introdução}

A insuficiência cardíaca tem sido apontada como o desfecho final da maioria das doenças cardiovasculares. $\mathrm{O}$ aumento da expectativa de vida da população e a otimização do tratamento do infarto agudo do miocárdio resultaram em aumento do número de indivíduos com insuficiência cardíaca ${ }^{1}$.

A incidência e a prevalência da insuficiência cardíaca tendem a aumentar progressivamente com o avanço da idade, o que faz com que seja considerada uma doença dos mais velhos. Após os 65 anos, a incidência da insuficiência cardíaca aproxima-se de 10 casos em mil, conforme dados de Thom et al. 2. Segundo a mesma fonte, $22 \%$ dos homens e $46 \%$ das mulheres vítimas de infarto do miocárdio, sofrerão das incapacidades advindas da insuficiência cardíaca após seis anos 2 .

A insuficiência cardíaca, uma vez instalada, tem forte impacto na vida do paciente, o que pode ser explicado pelas limitações decorrentes dos sintomas físicos e psicológicos associados à síndrome. Entre os sintomas físicos destacamse a fadiga e a dispnéia que se caracterizam pelo agravamento progressivo. Entre os sintomas emocionais destacam-se o medo, a insegurança e a tristeza. São ainda descritos, déficits de memória e de atenção, e diminuição do equilíbrio, o que pode predispor à ocorrência de quedas $3,4,5$. Esse conjunto de repercussões comprome- 
te a qualidade de vida relacionada à saúde dos sujeitos que a vivenciam, especialmente a do idoso.

Evangelista et al. ${ }^{6}$ relatam que a insuficiência cardíaca também está associada ao comprometimento do desempenho funcional - traduzido no relato dos idosos pelas limitações nas atividades habituais do dia-a-dia, incapacidade para o trabalho e no estabelecimento de relações sociais e perda da independência.

A independência funcional é definida como a capacidade de realizar algo com os próprios meios. Está ligada à mobilidade e à capacidade funcional, nas quais o indivíduo não requer ajuda para a realização das atividades de vida diária, ou seja, a independência supõe condições motoras e cognitivas satisfatórias para o desempenho destas tarefas 7 .

Assim, para os idosos uma qualidade de vida relacionada à saúde satisfatória pode ser interpretada como a possibilidade de conseguir cumprirsuasfunções diárias básicas adequadamente, se sentir bem e viver de forma independente 8,9 . Giatti \& Barreto 10, em estudo sobre saúde, trabalho e envelhecimento no Brasil, concluíram que a saúde, e em especial os indicadores de autonomia e mobilidade física, são fatores preditivos independentes da permanência na vida ativa em idades mais avançadas.

A avaliação da independência funcional em idosos tem sido considerada como um indicativo da qualidade de vida relacionada à saúde, uma vez que o desempenho nas atividades da vida diária permite aos profissionais da área da saúde uma visão ampla do comprometimento da saúde e seqüelas das doenças 9,11,12. Por outro lado, há na literatura estudos que evidenciam a manutenção de uma relativa qualidade de vida relacionada à saúde, a despeito da limitação funcional do idoso com insuficiência cardíaca 13,14,15.

Embora vários estudos apresentem relatos do comprometimento da qualidade de vida relacionada à saúde associado ao declínio da funcionalidade na insuficiência cardíaca, avaliada principalmente pela a Classificação Funcional da New York Heart Association (CF-NYHA) 16,17,18, são escassos os estudos que avaliaram a correlação entre a medida de qualidade de vida e de independência funcional 19 .

A demonstração dessa correlação tem implicações na avaliação e tratamento do idoso com insuficiência cardíaca, uma vez que pode subsidiar o desenho de intervenções que, por meio da melhora da independência funcional, possam resultar em melhoria de sua qualidade de vida relacionada à saúde.

Este estudo tem como objetivo verificar a correlação entre as medidas de qualidade de vida re- lacionada à saúde e de independência funcional em idosos portadores de insuficiência cardíaca.

\section{Método}

Participaram do estudo idosos portadores de insuficiência cardíaca, de ambos os sexos, com idade igual ou superior a 60 anos, que se encontravam em tratamento ambulatorial em dois hospitais de referência do Estado de São Paulo, Brasil. Foram excluídos do estudo pacientes com dificuldade de comunicação e expressão, e aqueles submetidos a transplante cardíaco, por tratar-se de um evento marcante na vida do sujeito, e os que se recusaram a participar do estudo de acordo com a Resolução $n^{\circ}$. 196/96.

O tamanho da amostra foi baseado no cálculo dos coeficientes de correlação de Spearman entre os escores (total e por dimensões) dos instrumentos - Minnesota Living With Heart Failure Questionnaire (LHFQ) e Medida de Independência Funcional (MIF) e entre os escores do LHFQ e MIF e CF-NYHA, obtidos em estudo piloto ( $\mathrm{n}=$ 25). Assim, para determinar coeficientes de correlação em torno de 0,30 , utilizando-se $\alpha=0,05$ e $\beta=0,20$, foi determinado o número mínimo de 89 sujeitos. Considerando-se que a coleta de dados ocorreu em hospitais de referência, com elevado número de atendimentos, optou-se por aumentar o número de sujeitos ( $\mathrm{n}=146)$, o que possibilitou maior representatividade da amostra e um maior poder do teste.

A técnica de amostragem deu-se por conveniência, ou seja, foram arrolados para o estudo todos os pacientes agendados para os dias de coleta que atenderam aos critérios de inclusão. Estes foram submetidos à entrevista individual privativa realizada pela própria pesquisadora, após a assinatura do Termo de Consentimento Livre e Esclarecido, com a aplicação dos seguintes instrumentos: (a) caracterização sócio-demográfica e clínica; (b) Minnesota Living With Heart Failure Questionnaire (LHFQ); (c) Medida de Independência Funcional (MIF).

\section{Caracterização sócio-demográfica e clínica}

Construído pelos pesquisadores com base na literatura e submetido à validade de conteúdo por meio da avaliação por juízes com reconhecido saber em cardiologia e geriatria. Foram estudadas variáveis sócio-demográficas - idade, sexo, estado civil, escolaridade, composição familiar, renda individual e familiar, e vínculo empregatício e variáveis clínicas - CF-NYHA, fração de ejeção do ventrículo esquerdo, número de condições clínicas associadas e de medicamentos em uso. A 
CF-NYHA foi obtida com base no registro da consulta médica realizada no mesmo dia da entrevista e a fração de ejeção do ventrículo esquerdo, por meio do resultado do ecodoplercardiograma ou cintilografia de primeira passagem, sendo classificada como normal ou rebaixada, de acordo com o valor de normalidade de cada método.

\section{Minnesota Living With Heart Failure Questionnaire (LHFQ)}

Instrumento específico de avaliação da qualidade de vida relacionada à saúde desenvolvido por Rector et al. 20, traduzido e validado para a língua portuguesa do Brasil por Carrara 21. O LHFQ é composto por 21 questões relativas às limitações que freqüentemente estão associadas com o quanto a insuficiência cardíaca impediu o paciente de viver como ele gostaria durante o último mês. As respostas às questões do LHFQ referem-se à percepção do próprio paciente com relação à influência da insuficiência cardíaca nos aspectos físicos, sócio-econômicos e psicológicos da vida. Para cada questão pode ser atribuído um valor de 0 (zero) a 5 (cinco), sendo o maior escore indicativo de pior qualidade de vida. O escore total é obtido por meio da soma dos 21 itens, com intervalo possível de 0 a 105 . O instrumento foi utilizado na sua íntegra conforme traduzido e validado por Carrara 21.

\section{Medida de Independência Funcional (MIF)}

É um dos métodos mais utilizados para a avaliação da funcionalidade e pode ser verificado por meio do auto-relato ou do desempenho do indivíduo nas atividades da vida diária. No presente estudo foi utilizado o auto-relato. O instrumento foi desenvolvido na década de 80, na tentativa de padronizar conceitos e definições sobre incapacidade e de obter um instrumento que permitisse uma avaliação global da reabilitação com base nas atividades cotidianas 22,23,24. Foi traduzido e validado para a cultura brasileira por Riberto et al. 25,26 . Trata-se de um conjunto de 18 tarefas, divididas em duas subescalas: (1) MIF motora (MIFm) - avalia as atividades referentes ao autocuidado, que compreende a avaliação do desempenho em relação à higiene matinal, banho, vestir-se acima e abaixo da cintura, uso do vaso sanitário; controle da urina e das fezes; transferências do leito para a cadeira/cadeira de rodas, para o vaso sanitário, para o chuveiro ou banheira e locomoção, com avaliação da marcha e do desempenho ao subir e descer escadas; (2) MIF cognitiva/social (MIFcs) - avalia a compreensão, expressão, interação social, resolução de problemas e memória.
Cada item pode ser classificado em uma escala de graus de dependência de 7 níveis, sendo que o valor 1 corresponde à dependência total $\mathrm{e}$ o valor 7 , independência completa. $\mathrm{O}$ paciente descreve como realiza cada tarefa e o avaliador pontua conforme o protocolo para aplicação do instrumento. $\mathrm{O}$ familiar/cuidador pode auxiliar na descrição da tarefa realizada, inclusive indicando quanto de ajuda o paciente necessita. A avaliação é feita pela soma da pontuação obtida nos 18 itens, com intervalo possível de 18 a 126 . Os maiores escores indicam maior independência funcional. O instrumento foi utilizado como proposto por Riberto et al. 25,26 sem modificação alguma.

Os dados obtidos foram submetidos às análises estatísticas descritiva, para tratamento dos dados sócio-demográficos e clínicos e escores de qualidade de vida relacionada à saúde e da MIF; de confiabilidade, com determinação do coeficiente alfa de Cronbach, para verificar a consistência interna dos itens dos instrumentos LHFQ e MIF. Foi estabelecido como evidência de consistência interna satisfatória um valor de alfa de Cronbach > 0,70 27 e de correlação - com emprego do coeficiente de correlação de Spearman para avaliar as correlações entre os escores (total e por dimensões/subescalas) do LHFQ e MIF e a CF-NYHA. A escolha do coeficiente de correlação de Spearman foi devido a amostra não obedecer a uma distribuição normal, necessitando, portanto, de testes não paramétricos. Para a interpretação da magnitude das correlações foi adotada a seguinte classificação dos coeficientes de correlação: coeficientes de correlação $<0,4$ (correlação de fraca magnitude), $\geq 0,4$ $\mathrm{a}<0,5$ (de moderada magnitude) $\mathrm{e} \geq 0,5$ (de forte magnitude) 28 .

Foram lançadas as seguintes hipóteses de correlação entre os instrumentos: (a) correlação inversa de moderada a forte magnitude - entre a dimensão física do LHFQ e subescala motora e total da MIF; dimensão emocional do LHFQ com a subescala cognitiva/social da MIF e MIFt e entre o escore total do LHFQ e todas as subescalas da MIF; (b) correlação inversa de fraca magnitude - entre a dimensão física do LHFQ e subescala cognitiva/social da MIF e dimensão emocional do LHFQ e subescala motora da MIF.

A direção dessas correlações se deve ao fato de que os escores do LHFQ são inversamente ordenados, ou seja, quanto maiores os escores, pior a qualidade de vida relacionada à saúde, $\mathrm{e}$ os escores da MIF são diretamente ordenados (maiores escores indicam maior independência funcional).

Foi utilizado um nível de significância de 5\% (p valor $<0,05$ ). 
O estudo foi aprovado pelas comissões de ética dos referidos serviços onde foi realizada a coleta de dados.

\section{Resultados}

O grupo de 146 idosos caracterizou-se por distribuição semelhante entre homens e mulheres, sendo $52 \%$ do sexo masculino, com média de idade de 68,6 $( \pm 6,9)$ anos e tempo médio de 3,4 $( \pm 2,8)$ anos de estudo. A maioria era aposentada $(80,8 \%)$, vivia com a família $(88,4 \%)$ e relatou renda média - individual e familiar - de 2,0 $( \pm 2,3)$ e 3,0 $( \pm 2,4)$ salários-mínimos, respectivamente.

A caracterização clínica evidenciou idosos com média de 3,9 $( \pm 1,7)$ co-morbidades, sintomáticos, em média $3,5( \pm 1,4)$ sintomas relatados, com destaque para fadiga e dispnéia, em CF-NYHA I e II (65\%), com fração de ejeção do ventrículo esquerdo rebaixada $(66,7 \%)$ e com consumo médio de $5,1( \pm 1,9)$ medicamentos ao dia. A caracterização sócio-demográfica e clínica dos sujeitos estudados está apresentada na Tabela 1 .

Na Tabela 2 estão apresentados os escores obtidos com a aplicação do LHFQ (total e por dimensões), os escores da MIF (total e por subescalas), bem como os valores do alfa de Cronbach para ambos os instrumentos.

A pontuação observada com a aplicação da MIF mostrou escores médios elevados, principalmente na pontuação total, o que aponta para independência funcional dos sujeitos estudados. Os resultados obtidos com a aplicação do LHFQ mostraram que a mediana dos escores nas diferentes dimensões para este grupo de pacientes esteve próxima da média do valor máximo que poderia ser obtido em todas as dimensões, sendo que, as médias, embora próximas, apresentaram amplos desvios - padrão, indicando maior variabilidade dos escores.

A avaliação da consistência interna de ambos os instrumentos indicou homogeneidade dos itens, com menor valor do alfa de Cronbach para o LHFQ emocional $(\alpha=0,63)$.

A Tabela 3 apresenta a média dos escores da MIF e LHFQ dos idosos estudados de acordo com a CF-NYHA. As médias dos escores da MIF e do LHFQ em relação às classes funcionais mostraram que, com a progressão na CF-NYHA, os escores da MIF diminuem (nas subescalas e no escore total) e os escores do LHFQ (total e por dimensões) aumentam.

A Tabela 4 aponta os valores do coeficiente de correlação de Spearman entre os escores da MIF e do LHFQ. O sentido inverso das correlações entre as variáveis confirma que há relações inversas entre qualidade de vida relacionada à saúde e independência funcional, uma vez que o LHFQ é inversamente ordenado (maiores valores, pior qualidade de vida relacionada à saúde) e a MIF é diretamente ordenada (maiores valores, maior independência funcional).

Foram constatadas correlações significantes de moderada magnitude entre as dimensões física do LHFQ e a MIFm $(-0,49)$, e MIFt $(r=-0,47)$ e de fraca magnitude entre esta dimensão e a MIFcs $(r=-0,26)$. A dimensão emocional do LHFQ apresentou correlação significante de fraca magnitude com a MIFm $(r=-0,34)$ e MIFt $(r=-0,36)$. Também foi fraca a correlação entre a dimensão emocional do LHFQ e a MIFcs $(r=-0,29)$, contrariando a hipótese de forte correlação entre domínios similares.

O escore total do LHFQ apresentou correlações significantes de moderada magnitude com a MIFm $(r=-0,44)$ e MIFt $(r=-0,43)$, e de fraca magnitude com a MIFcs $(r=-0,26)$.

Para avaliar o comportamento da correlação entre as duas medidas - qualidade de vida relacionada à saúde e independência funcional - nas diferentes classes funcionais da insuficiência cardíaca, foi reproduzida a análise de correlação para cada um dos grupos da CF-NYHA. Pelo fato da classe IV ser composta por pequeno número de sujeitos, optou-se pelo agrupamento das classes III e IV, dando origem à classe III/IV (Tabela 5).

Os dados indicam que na CF-NYHA I, todas as subescalas da MIF foram inversamente correlacionadas com as dimensões do LHFQ. O maior valor de correlação observado foi entre a MIFm e LHFQf $(r=-0,50)$. Não foram observadas correlações entre qualidade de vida relacionada à saúde e MIF em pacientes em CF-NYHA II. Na CF-NYHA III/IV foram encontradas correlações significantes e de fraca magnitude entre a MIFm e LHFQ físico $(r=-0,36)$ e LHFQt $(r=-0,36)$, e entre MIFt e LHFQf $(r=-0,31)$ e LHFQt $(r=-0,32)$.

A Figura 1 ilustra o comportamento do escore total do LHFQ e MIF em relação à CF-NYHA, indicando que, à medida que a independência funcional aumenta, melhora a qualidade de vida relacionada à saúde.

\section{Discussão}

Neste estudo, os idosos portadores de insuficiência cardíaca de menor gravidade (CF-NYHA I e II) apresentaram escores de qualidade de vida relacionada à saúde intermediários em todas as dimensões do LHFQ, indicando moderado comprometimento da qualidade de vida relacionada à saúde, a exemplo de estudos pregressos 17,18,29,30. Da mesma forma, as médias dos escores da MIF 
Caracterização sócio-demográfica e clínica dos 146 idosos estudados. São Paulo, Brasil, 2005.

\begin{tabular}{|c|c|c|c|c|}
\hline Variável & n (\%) & Média \pm DP & Mediana & Variação observada \\
\hline Idade (anos) & & $68,6 \pm 6,9$ & 67 & $60-87$ \\
\hline \multicolumn{5}{|l|}{ Sexo } \\
\hline Masculino & $76(52,0)$ & & & \\
\hline Feminino & $70(47,9)$ & & & \\
\hline Escolaridade (anos) & & $3,4 \pm 2,8$ & 4 & $0-20$ \\
\hline Renda pessoal (salários mínimos) * & & & 1 & $0-20$ \\
\hline Pessoal & & $2,0 \pm 2,3$ & 3 & $0-20$ \\
\hline Familiar & & $3,0 \pm 2,4$ & & \\
\hline \multicolumn{5}{|l|}{ Vínculo empregatício } \\
\hline Aposentado & $118(80,8)$ & & & \\
\hline Ativo & $8(5,5)$ & & & \\
\hline Auxílio doença & $4(2,7)$ & & & \\
\hline Pensionista & $2(1,4)$ & & & \\
\hline Do lar & $14(9,6)$ & & & \\
\hline \multicolumn{5}{|l|}{ Composição familiar } \\
\hline Mora sozinho & $17(11,6)$ & & & \\
\hline Mora com família/Companheiro/Filhos & $129(88,4)$ & & & \\
\hline Número de co-morbidades & & $3,9 \pm 1,7$ & & \\
\hline $1-2$ & $32(21,9)$ & & & \\
\hline $3-4$ & $54(37,0)$ & & & \\
\hline $5-6$ & $50(34,2)$ & & & \\
\hline$>6$ & $10(6,8)$ & & & \\
\hline Sintomas & & $3,5 \pm 1,4$ & & \\
\hline Fadiga & $120(82,2)$ & & & \\
\hline Dispnéia & $113(77,4)$ & & & \\
\hline Precordialgia & $67(45,9)$ & & & \\
\hline Tosse & $64(43,8)$ & & & \\
\hline \multicolumn{5}{|l|}{ CF-NYHA } \\
\hline I & $49(33,5)$ & & & \\
\hline II & $46(31,5)$ & & & \\
\hline III & $42(28,8)$ & & & \\
\hline IV & $9(6,2)$ & & & \\
\hline Fração de ejeção do ventrículo esquerdo & & $0,46 \pm 0,17$ & & \\
\hline Rebaixada & $90(66,7)$ & & & \\
\hline Normal & $45(33,3)$ & & & \\
\hline Número de medicamentos & & $5,1 \pm 1,9$ & & \\
\hline $1-3$ & $27(18,5)$ & & & \\
\hline $4-6$ & $81(55,5)$ & & & \\
\hline $7-9$ & $36(24,6)$ & & & \\
\hline$>10$ & $2(1,4)$ & & & \\
\hline
\end{tabular}

CF-NYHA: Classificação Funcional da New York Heart Association.

* Salário mínimo $=$ US\$113,00.

e do LHFQ em relação à CF-NYHA demonstraram que, com a piora funcional da insuficiência cardíaca os valores da MIF diminuem e do LHFQ aumentam.

Foram constatados valores do alfa de Cronbach acima de 0,70 para ambos os instrumentos 27. Em relação à MIF foram constatados valores de alfa de Cronbach entre 0,80 e 0,90, a exemplo de estudos prévios 12,31. Quanto ao LHFQ foram observados valores satisfatórios na dimensão física e escore total abaixo do critério satisfatório na dimensão emocional. Estudos anteriores que avaliaram a consistência interna do LHFQ também evidenciaram valores do al- 
Estatística descritiva e confiabilidade do Minnesota Living With Heart Failure Questionnaire (LHFQ, total e por dimensões) e da Medida de Independência Funcional (MIF, total e subescalas) para os 146 idosos com insuficiência cardíaca. São Paulo, Brasil, 2005.

\begin{tabular}{|c|c|c|c|c|c|c|}
\hline Variável & Número de itens & Média \pm DP & Mediana & Variação observada & Variação possível & Alfa de Cronbach \\
\hline \multicolumn{7}{|c|}{ LHFQ (dimensões) } \\
\hline LHQf & 21 & $18,7 \pm 9,1$ & 19,0 & $0-40$ & $0-40$ & 0,80 \\
\hline LHFQe & 8 & $10,2 \pm 5,8$ & 10,5 & $0-22$ & $0-25$ & 0,63 \\
\hline LHFOt & 5 & $41,3 \pm 17,8$ & 41,0 & $2-91$ & $0-105$ & 0,82 \\
\hline \multicolumn{7}{|c|}{ MIF (subescalas) } \\
\hline MIFm & 13 & $70,8 \pm 9,7$ & 72,0 & $27-88$ & $13-91$ & 0,90 \\
\hline MIFcs & 5 & $25,6 \pm 4,4$ & 25,0 & $15-35$ & $5-35$ & 0,73 \\
\hline MIF t & 18 & $96,4 \pm 12,6$ & 96,5 & $46-121$ & $18-126$ & 0,90 \\
\hline
\end{tabular}

LHFQf: dimensão física; LHFQe: dimensão emocional; LHFQt: escore total; MIFm: subescala motora; MIFcs: subescala cognitiva/social; MIFt: escore total.

Médias dos escores da Medida de Independência Funcional (MIF, total e subescalas) e do Minnesota Living With Heart Failure Questionnaire (LHFQ, total e dimensões), segundo a Classificação Funcional da New York Heart Association (CF-NYHA) dos 146 idosos com insuficiência cardíaca. São Paulo, Brasil, 2005.

\begin{tabular}{|c|c|c|c|c|}
\hline & \multicolumn{4}{|c|}{ CF-NYHA } \\
\hline & $I(n=49)$ & II $(n=46)$ & III $(n=42)$ & $\mathrm{IV}(\mathrm{n}=9)$ \\
\hline & Média \pm DP & Média \pm DP & Média \pm DP & Média \pm DP \\
\hline \multicolumn{5}{|c|}{ MIF (subescalas) } \\
\hline $\mathrm{MIFm}$ & $76,2 \pm 6,9$ & $70,4 \pm 9,4$ & $68,6 \pm 6,8$ & $53,8 \pm 12,5$ \\
\hline MIFCS & $27,4 \pm 4,5$ & $24,8 \pm 4,4$ & $24,2 \pm 3,7$ & $26,4 \pm 2,9$ \\
\hline MIFt & $103,6 \pm 10,6$ & $95,3 \pm 12,4$ & $92,9 \pm 9,4$ & $80,2 \pm 14,5$ \\
\hline \multicolumn{5}{|c|}{ LHFQ (dimensões) } \\
\hline LHOf & $12,7 \pm 8,3$ & $18,6 \pm 7,0$ & $22,5 \pm 7,3$ & $33,5 \pm 3,2$ \\
\hline LHFQe & $7,8 \pm 6,1$ & $9,8 \pm 4,5$ & $12,0 \pm 5,6$ & $16,1 \pm 5,0$ \\
\hline LHFQt & $31,1 \pm 16,8$ & $39,6 \pm 12,5$ & $47,7 \pm 14,0$ & $74,7 \pm 7,9$ \\
\hline
\end{tabular}

MIFm: subescala motora; MIFcs: subescala cognitiva/social; MIFt: escore total; LHFQf: dimensão física; LHFQe: dimensão emocional; LHFQt: escore total.

fa de Cronbach inferiores àqueles encontrados na dimensão física e escore total, porém nestes estudos os valores encontrados foram superiores a 0,80 . Segundo Streiner \& Norman 32 , o valor do alfa de Cronbach pode ser influenciado pela correlação dos itens de uma medida e também pelo número de itens que compõe a escala 18,30,33. Dessa forma, o pequeno número de itens da subescala emocional pode ter contribuído para o valor do alfa de Cronbach inferior ao desejado.

A avaliação funcional tem sido destacada pela literatura como um importante conceito para a compreensão do impacto da insuficiência cardíaca no dia-a-dia dos pacientes. Inclui uma variedade de fatores, tais como a percepção dos sintomas, barreiras ambientais, neces- sidade de ajuda e apoio social, bem como fatores psicológicos, que são todos relatados pelos pacientes.

Embora qualidade de vida relacionada à saúde e status funcional sejam conceitos potencialmente correlacionados, representam diferentes componentes da condição de saúde de um indivíduo. O resultado da avaliação funcional é uma medida das habilidades para realização de tarefas específicas sem significante limitação física, e mais recentemente vem emergindo como um componente-chave para a avaliação da saúde dessa população ${ }^{34}$. Por outro lado, a qualidade de vida relacionada à saúde é um conceito que reflete a discrepância entre a percepção do paciente em relação ao seu estado de saúde atual daquele esperado, ou seja, a qualidade de vida 
Valores do coeficiente de correlação de Spearman entre os escores do Minnesota Living With Heart Failure Questionnaire (LHFQ) e da Medida de Independência Funcional (MIF), obtidos junto aos 146 idosos com insuficiência cardíaca. São Paulo, Brasil, 2005.

\begin{tabular}{lcccc}
\hline & MIFm & MIFcs & MIFt & LHFQf \\
\hline MIFcs & $0,57 *$ & & & \\
MIFt & $0,95 *$ & $0,77 *$ & & \\
LHFQf & $-0,49 * *$ & $-0,26 * \star$ & $-0,47 * *$ & $0,52 *$ \\
LHFQe & $-0,34 * *$ & $-0,29 *$ & $-0,36 * *$ & $0,89 *$ \\
LHFQt & $-0,44 * *$ & $-0,26 * * *$ & $-0,43 * *$ & $0,73 *$ \\
\hline
\end{tabular}

MIFm: subescala motora; MIFcs: subescala cognitiva/social; MIFt: escore total; LHFQf: dimensão física; LHFQe: dimensão emocional; LHFQt: escore total.

$* p<0,01$.

${ }^{* *} p=0,0001$

$\star \star \star ~ p=0,001$

$\# p=0,0002$.

Valores do coeficiente de correlação de Spearman entre os escores do Minnesota Living With Heart Failure Questionnaire (LHFQ) e da Medida de Independência Funcional (MIF) para cada classe da Classificação Funcional da New York Heart Association (CF-NYHA), obtidos junto a 146 idosos com insuficiência cardíaca.

\begin{tabular}{|c|c|c|c|c|c|c|c|c|c|}
\hline & \multicolumn{9}{|c|}{ CF-NYHA } \\
\hline & \multicolumn{3}{|c|}{$I(n=49)$} & \multicolumn{3}{|c|}{ II $(n=46)$} & \multicolumn{3}{|c|}{ III/IV (n = 51) } \\
\hline & MIFm & MIFcs & MIFt & MIFm & MIFcs & MIFt & MIFm & MIFcs & MIFt \\
\hline \multirow[t]{2}{*}{ LHFOf } & $-0,50$ & $-0,37$ & $-0,49$ & $-0,18$ & $-0,12$ & $-0,19$ & $-0,36$ & $-0,05$ & $-0,31$ \\
\hline & $(p=0,0002)$ & $(p=0,007)$ & $(p=0,003)$ & $(p=0,22)$ & $(p=0,42)$ & $(p=0,20)$ & $(p=0,008)$ & $(p=0,72)$ & $(p=0,02)$ \\
\hline \multirow[t]{2}{*}{ LHFQe } & $-0,40$ & $-0,44$ & $-0,43$ & $-0,16$ & $-0,25$ & $-0,23$ & $-0,21$ & $-0,04$ & $-0,17$ \\
\hline & $(p=0,003)$ & $(p=0,001)$ & $(p=0,002)$ & $(p=0,27)$ & $(p=0,08)$ & $(p=0,12)$ & $(p=0,12)$ & $(p=0,77)$ & $(p=0,212)$ \\
\hline \multirow[t]{2}{*}{ LHFQt } & $-0,45$ & $-0,35$ & $-0,42$ & $-0,15$ & $-0,14$ & $-0,186$ & $-0,36$ & $-0,004$ & $-0,32$ \\
\hline & $(p=0,001)$ & $(p=0,013)$ & $(p=0,002)$ & $(p=0,30)$ & $(p=0,34)$ & $(p=0,21)$ & $(p=0,008)$ & $(p=0,97)$ & $(p=0,019)$ \\
\hline
\end{tabular}

MIFm: subescala motora; MIFcs: subescala cognitiva/social; MIFt: escore total; LHFQf: dimensão física; LHFQe: dimensão emocional; LHFQt: escore total.

relacionada à saúde é melhor avaliada quando há maior concordância entre a percepção do paciente de sua saúde atual e a esperada 15 .

$\mathrm{Na}$ literatura encontram-se estudos que investigaram a correlação entre a qualidade de vida relacionada à saúde (medida por meio do LHFQ) em pacientes com insuficiência cardíaca e a sua funcionalidade avaliada por meio de outras medidas de desempenho. O estudo de Gorkin et al. 35 , por exemplo, avaliou o desempenho dos indivíduos por meio do The 6-Minute Walk Test e pela Functional Status Scale (physical limitations) e obteve correlações de 0,39 e 0,75, respectivamen- te. Heo et al. 30 constataram correlação positiva entre o nível da Specific Activity Scale (um método para avaliar status funcional com base no custo metabólico por atividades) e a dimensão física do LHFQ $(r=0,52)$ e o escore total do LHFQ $(\mathrm{r}=0,41)$ e correlação de fraca magnitude com a dimensão emocional $(0,28)$. No estudo de Demers et al. 36, o The 6-Minute Walk Test foi fracamente e inversamente correlacionado com o escore de qualidade de vida relacionada à saúde $(r=-0,26)$.

No entanto, não foram encontrados estudos sobre a correlação da MIF e qualidade de vida 
Figura 1

Correlação entre os escores totais do Minnesota Living With Heart Failure Questionnaire (LHFQ) e da Medida de Independência Funcional (MIF), de acordo com as classes I, II, III e IV da Classificação Funcional da New York Heart Association (CF-NYHA), em idosos com insuficiência cardíaca.

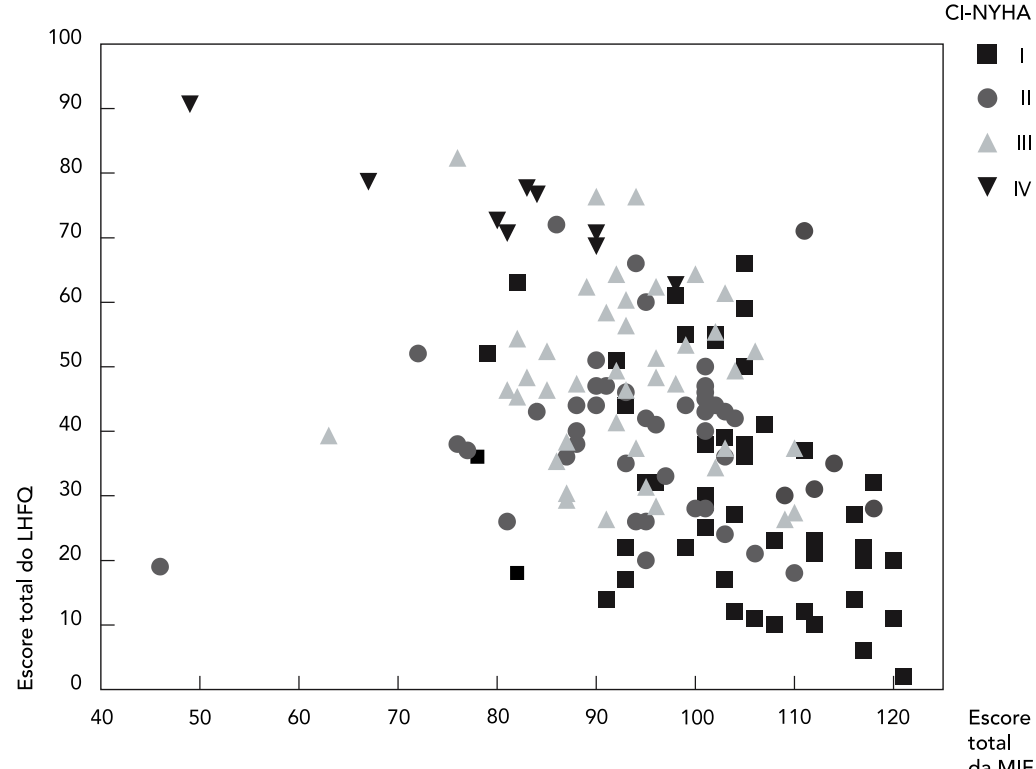

relacionada à saúde em pacientes com insuficiência cardíaca. Há na literatura relatos de estudos que avaliaram a correlação entre a MIF e a medida da qualidade de vida relacionada à saúde, mensurada por diferentes instrumentos na presença de outras doenças. O estudo de Cruz 12 avaliou a relação entre a MIF e a qualidade de vida relacionada à saúde em pacientes com acidente vascular encefálico. Foi encontrada correlação significante entre MIFm, MIFcs e MIFt e o domínio capacidade funcional da medida de qualidade de vida relacionada à saúde, obtida com emprego do $S F-36$ - um instrumento multidimensional de avaliação do estado geral de saúde composto por 36 itens que medem: capacidade funcional, aspecto físico, aspectos emocionais, saúde mental e inclui também uma questão de avaliação comparativa entre as condições de saúde atual e as que antecedem um ano da entrevista. Rockwood et al. 19, em estudo de revisão para comparar as diversas medidas utilizadas na avaliação das mudanças no estado de saúde de idosos frágeis, relataram correlação de moderada magnitude entre a MIF e o Índice de Qualidade de Vida de Spitzer $(\mathrm{r}=0,45)$. Be- thoux et al. 37 estudaram a relação entre a MIF e o Reintegration to Normal Living Index em pacientes com hemiplegia, e observaram que a qualidade de vida relacionada à saúde pode deteriorar em alguns domínios, mesmo quando o nível de incapacidade não modifica.

Algumas das hipóteses de correlações inversas - entre a MIF e a medida de qualidade de vida relacionada à saúde - lançadas neste estudo foram parcialmente confirmadas. Eram esperadas correlações de moderada a forte magnitude entre subescalas/dimensões similares. Embora tal hipótese tenha se confirmado para a dimensão física do LHFQ e MIFm ( $\mathrm{r}=-0,49)$, LHFQ total e MIFm $(r=-0,44)$, LHFQ total e MIFt $(r=-0,43)$, foi fraca a magnitude da correlação observada entre a dimensão emocional do LHFQ e MIFcs $(\mathrm{r}=-0,29)$, e LHFQ total e MIFcs $(\mathrm{r}=-0,26)$.

Tais achados indicam que as subescalas da MIF estão relacionadas à qualidade de vida relacionada à saúde, especialmente no que se refere à dimensão física e escore total. A fraca correlação observada entre a dimensão emocional do LHFQ com a MIFcs, pode ser explicada pela discrepância conceitual dos dois instrumentos, no que se refere ao aspectos emocionais. Enquanto a dimensão emocional do LHFQ busca avaliar a carga emocional representada pela insuficiência cardíaca, a subescala cognitiva/social da MIF avalia o desempenho do sujeito em relação à compreensão, expressão, interação social, resolução de problemas e memória.

A análise das correlações entre as medidas de qualidade de vida relacionada à saúde e MIF, de acordo com a CF-NYHA, evidencia que as correlações entre as duas medidas se mantiveram somente para aqueles classificados nos extremos da CF-NYHA, ou seja, nas classes I e III/IV.

Os resultados das análises de correlação entre MIF e qualidade de vida relacionada à saúde nas diferentes classes da CF-NYHA, embora devam ser interpretados com limitação, devido ao pequeno número de sujeitos na classe IV, sugerem que a independência funcional somente se correlaciona com qualidade de vida relacionada à saúde nos estratos formados pelos extremos da CF-NYHA.

Foram observadas correlações de maior magnitude na CF-NYHA I em comparação às observadas na classe III/IV, o que pode estar relacionado com a variabilidade dos escores nas respectivas classes. A ausência de correlação nos grupos intermediários da CF-NYHA deve ser investigada em estudos futuros, eventualmente com ampliação da amostra e com emprego de outras técnicas de análise, que não somente a correlação. 


\section{Considerações finais}

Os achados deste estudo evidenciam correlações de moderada magnitude entre a subescala motora e escore total da MIF e todas as dimensões do LHFQ, o que sugere que medidas que promovam a independência funcional podem levar à melhor qualidade de vida relacionada à saúde, especialmente no domínio físico. Novos estudos devem ser conduzidos envolvendo maior número de sujeitos com maior severidade da doença para verificar a reprodutibilidade destes achados.

\section{Resumo}

Este estudo teve como objetivo identificar a correlação entre as medidas de qualidade de vida relacionada à saúde e independência funcional em idosos com insuficiência cardíaca. Fizeram parte do estudo 146 idosos com insuficiência cardíaca em tratamento ambulatorial, que responderam ao Minnesota Living With Heart Failure Questionnaire (LHFQ) e à Medida de Independência Funcional (MIF). Foi utilizado o coeficiente de correlação de Spearman para verificar a correlação entre as medidas de qualidade de vida relacionada à saúde e MIF, e entre estas e a Classificação Funcional da New York Heart Association (CF-NYHA). Foram constatadas correlações significantes de moderada magnitude entre as dimensões física e emocional do LHFQ e as subescalas total e motora da MIF. Os resultados evidenciaram que a qualidade de vida relacionada à saúde e independência funcional são conceitos correlacionados e influenciados pela CF-NYHA. Os resultados sugerem que ações voltadas à promoção da independência funcional podem otimizar a qualidade de vida relacionada à saúde de idosos com insuficiência cardíaca, especialmente na dimensão física. São necessários novos estudos com ampliação da amostra, inclusão de pacientes de faixa etária mais elevada e com maior gravidade da doença, para verificar a reprodutibilidade destes achados.

Insuficiência Cardíaca Congestiva; Atividades Cotidianas; Qualidade de Vida; Idoso

\section{Colaboradores}

F. A. A. Scattolin participou da revisão de literatura, da elaboração da metodologia, análise dos resultados e redação do artigo final. M. J. D’E. Diogo e R. C. R. Colombo colaboraram na elaboração da metodologia, análise dos resultados e redação do artigo final. 


\section{Referências}

1. Remme WJ, McMurray JJV, Rauch B, Zannad F, Keukelaar K, Cohen-Solal A, et al. Public awareness of heart failure in Europe: first result from SHAPE. Eur Heart J 2005; 26:2413-21.

2. Thom T, Haase N, Rosamond W, Howard VJ, Rumsfeld J, Manolio T, et al. Heart disease and stroke statistics-2006 update: a report from the American Heart Association Statistics Committee and Stroke Statistics Subcommittee. Circulation 2006; 113:e85-151.

3. Dracup K, Walden JA, Stevenson LW, Brecht M. Quality of life with advanced heart failure. J Heart Transplant 1992; 11:273-9.

4. Belardinelli R, Demetrios G, Giovani C, Purcaro A. Randomized controlled trial of long-term moderate exercise training in chronic heart failure: effects on functional capacity, quality of life, and clinical outcome. Circulation 1999; 99:1173-82.

5. Struthers AD. Heart failure: the diagnosis of heart failure. Heart 2000; 84:334-8.

6. Evangelista LS, Kagawa-Singer M, Dracup K. Gender differences in health perception and meaning in person living with heart failure. Heart Lung 2001; 30:167-76.

7. Neri AL. Palavras-chave em gerontologia. Campinas: Editora Alínea; 2001.

8. Spidurso WW, Cronin DL. Exercise-dose response effects on quality of life and independent living in older adults. Med Sci Sports Exerc 2001; 33(6 Suppl):S598-608.

9. Paschoal SMP. Autonomia e independência. In: Papaléo Netto M, organizador. Gerontologia: a velhice e o envelhecimento em visão globalizada. São Paulo: Editora Atheneu; 2002. p. 311-23.

10. Giatti L, Barreto SM. Saúde, trabalho e envelhecimento no Brasil. Cad Saúde Pública 2003; 19:75971.

11. Paula JAM, Tavares MCGDF, Diogo MJD'E. Avaliação funcional em gerontologia. Gerontologia 1998; 6:81-8.

12. Cruz KCT. Avaliação da capacidade funcional e da qualidade de vida de indivíduos com acidente vascular encefálico com idade maior ou igual a 50 anos [Dissertação de Mestrado]. Campinas: Universidade Estadual de Campinas; 2004.

13. Albrecht GL, Devlieger PJ. The disability paradox: high quality of life against all odds. Soc Sci Med 1999; 48:977-88.

14. Levenson JW, McCarthy EP, Lynn J, Davis RB, Phillips RS. The last six months of life for patients with congestive heart failure. J Am Geriatr Soc 2000; 48:101-9.

15. Masoudi FA, Rumsfeld JS, Havranek EP, House JA, Peterson ED, Krumholz HM, et al. Age, functional capacity, and health-related quality of life in patients with heart failure. J Cardiac Fail 2004; 10:368-73.

16. Carels RA. The association between disease severity, functional status, depression and daily quality of life in congestive heart failure patients. Qual Life Res 2004; 13:62-73

17. Riegel B, Moser DK, Glaser D, Carlson B, Deaton C, Armola R. The Minnesota Living With Heart Failure Questionnaire. Nurs Res 2002; 51:209-18.
18. Bennett SJ, Oldridge NB, Eckert GJ, Embree JL, Browning S, Hou N, et al. Discriminant properties of commonly used quality of life measures in heart failure. Qual Life Res 2002; 11:349-59.

19. Rockwood K, Stolee P, Fox RA. Use of goal attainment scaling in measuring clinically important change in the frail elderly. J Clin Epidemiol 1993; 46:1113-8.

20. Rector TS, Kubo SH, Cohn JN. Patients' self-assessment of their congestive failure. Part 2 - content, reliability and validity of a measure, the Minnesota Living With Heart Failure Questionnaire. Heart Fail 1987; 3:198-209.

21. Carrara D. Avaliação prospectiva da qualidade de vida em pacientes com miocardiopatia dilatada submetidos a ventriculectomia parcial esquerda [Dissertação de Mestrado]. São Paulo: Universidade de São Paulo; 2001.

22. Granger CV, Hamilton BB, Keith RA, Zielezny M, Sherwin FS. Advances in functional assessment for medical rehabilitation. Topics in Geriatric Rehabilitation 1986; 1:59-74.

23. Ottenbacher KJ, Hsu Y, Granger CV, Fiedler RC. The reliability of the functional independence measure: a quantitative review. Arch Phys Med Rehabil 1996; 77:1226-32.

24. Cohen ME, Marino RJ. The tools of disability outcomes research functional status measures. Arch Phys Med Rehabil 2000; 81:21-9.

25. Riberto M, Miyasaki HM, Jorge Filho D, Sakamoto H, Battistella LR. Reprodutibilidade da versão brasileira de Medida de Independência Funcional. Acta Fisiátrica 2001; 8:45-52.

26. Riberto M, Miyasaki HM, Jucá SHH, Sakamoto H, Pinto PPN, Battistella LR. Validação da versão brasileira da Medida de Independência Funcional. Acta Fisiátrica 2004; 11:72-6.

27. Lobiondo-Wood G, Haber J. Confiabilidade e validade. In: Lobiondo-Wood G, Haber J, organizadores. Pesquisa em enfermagem: métodos, avaliação crítica e utilização. 3a Ed. Rio de Janeiro: Editora Guanabara Koogan; 2001. p. 186-99.

28. Hulley SB, Cummings SR, Browner WS, Grady D, Hearst N, Newman TB. Delineando a pesquisa clínica: uma abordagem epidemiológica. 2a Ed. Porto Alegre: Editora Artmed; 2003.

29. Calvert MJ, Freemantle N, Cleland JGF. The impact of chronic heart failure on Health-related Quality of Life data acquired in the baseline phase of the CARE-HF Study. Eur J Heart Fail 2005; 7:243-51.

30. Heo S, Moser DK, Riegel B, Hall LA, Christman N. Testing the psychometric properties of Minnesota Living With Heart Failure Questionnaire. Nurs Res 2005; 54:265-72.

31. Stineman MG, Shea JA, Jette A, Tassoni CJ, Ottenbacher KJ, Fidler R, et al. The functional independence measure: tests of scaling assumptions, structure, and reliability across 20 diverse impairment categories. Arch Phys Med Rehabil 1996; 77:1101-8.

32. Streiner DL, Norman GR. Health measurement scales: a practical guide to their development and use. $2^{\text {nd }}$ Ed. New York: Oxford University Press; 2001. 